\title{
Agenesis of all third molars in two half siblings
}

Jamie A. Kaufer ${ }^{1}$

${ }^{1}$ University of Pittsburgh School of Dental Medicine, Pittsburgh PA, USA

\section{Abstract}

Tooth agenesis is one of the most common dental anomalies and is influenced by factors including patient genetics. Although there are several specific genes associated with certain patterns of agenesis, there does not seem to be a genetic pattern identified to date that is associated with isolated complete third molar agenesis. This report presents two half-siblings who both express complete agenesis of third molars despite the fact that their shared parent does not express the same phenotype. The case discussion focuses on addressing the potential genetic possibilities including autosomal dominant inheritance and variable expressivity of a mutation. There remains an uncertainty to the exact gene involved. Potential options include WNT1OA and PAX9. The affected individuals are not needing extractions or experiencing complications due to the presence of third molars. However, the concern remains that there is the potential for more severe expression of the mutation in future generations.

\section{Introduction}

The agenesis of teeth has been continuously reported as one of the most common dental abnormalities in humans. The classifications of missing teeth are in three categories: hypodontia is defined as missing one to six teeth, oligodontia meaning missing more than six teeth, and anodontia missing all teeth. The definitions of both hypodontia and oligodontia, however, exclude third molars (commonly known as wisdom teeth). The agenesis of third molars is a very common, if not the most common, pattern of tooth agenesis [1]. Many genes have been associated with tooth development [2]. Certain genes, including $P A X 9, M S X 1, A X I N 2$, EDA, EDAR, EDARADD, and WNT10A have been associated with specific patterns of tooth loss. However most instances of tooth agenesis are not associated with any of these genes. PAX9 has been associated with third molar agenesis, but no coding mutations in that gene have been identified that explain isolated third molar agenesis [3]. This report describes a case of third molar agenesis between two half-siblings followed by a discussion of potential genetic influence.

\section{Case Report}

A 24-year old Caucasian female reported to the University of Pittsburgh School of Dental Medicine for routine care. Upon taking radiographs, it was observed that her third molars were not present. The patient reported that her third mo- lars were not extracted and had never erupted. When asked further, the patient stated that her halfbrother, 15 years old, was also missing all of his third molars, and that all parents of the siblings had at least two wisdom teeth. Regarding the pedigree (Figure 1), the only information that could be completed was of the immediate parents. Individual 2.5 , the mother of individual 3.4, had two wisdom teeth, both in the maxillary arch and fully erupted. Individual 2.3, the mother of individual 3.3, had all four wisdom teeth removed. Individual 2.4, the father of both individuals 3.3 and 3.4, had three third molars removed. No known genetic testing for any individuals in the family regarding tooth agenesis has been completed. It should be noted that individual 3.3 is missing his maxillary first premolars as well, but the- (cc) BY ULLS D-dorle
New articles in this journal are licensed under a Creative Commons Attribution 4.0 United States License.

This journal is published by the University Library System, University of Pittsburgh as part of its D-Scribe Digital Publishing Program and is cosponored by the University of Pittsburgh Press. 
1

3

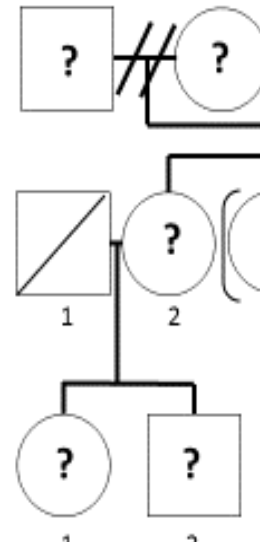

1

2
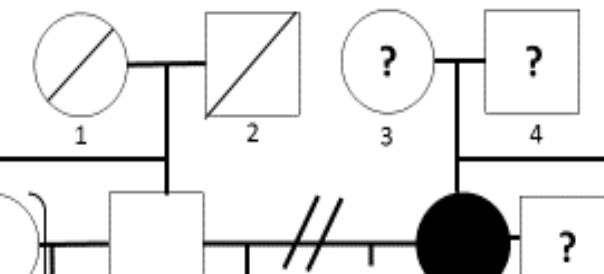

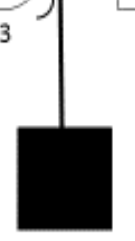

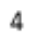

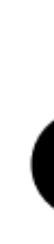

Figure 1: Family pedigree of agenesis of all third molars

se were extracted in preparation for orthodontic treatment.

\section{Discussion}

In most research regarding tooth agenesis, third molars are often excluded from the studies. It is for that reason that it is difficult to discuss the exact potential genetic cause of agenesis of third molars. For this particular case, there seems to be some history of missing third molars for individual 3.4 in both of her parents. However, neither parent is missing all four of their third molars. Individual 3.3 has only one parent, individual 2.4, who is missing any third molars. However, despite the difference in parental presence of third molars, the same phenotype resulted in individuals 3.3 and 3.4.

As mentioned previously, individual 2.5 is missing both third molars in the mandible, which suggests a particular pattern of agenesis associated with a genetic influence. For individual 3.4, there is a chance that given one parent expresses a phenotype of partial agenesis that her phenotype of complete agenesis is the result of an inherited mutation with more severe expression. As for individual 3.3 , there could be a chance of inherited mutation from individual 2.4 or 2.3 , or a spontaneous mutation.

Considering individuals 3.3 and 3.4 have the same phenotype and only one shared parent, there is also the potential that there may be a pattern of inheritance in this family. There is a good chance that this case follows an autosomal dominant pattern of inheritance. Using the pedigree as a reference, it is unlikely that whatever genetic mutation took place is $\mathrm{x}$-linked because there is not a preference for a particular biological sex. It is also unlikely that this mutation followed an autosomal recessive pattern of inheritance. In order for this case to be recessive, multiple people in the family would be carriers of the gene, which would be very unlikely.

There is also the question of which genes may be involved in the agenesis molars in this case. WNT10A mutations are the most common identifiable cause of hypo- dontia. PAX9, a homeobox gene on chromosome 14 , has also been associated with preferential agenesis of the molars due to haploinsufficiency. Haploinsufficiency means that missing one copy of the gene decreased the amount of translated protein, therefore increasing the chance of missing teeth [4]. Another possibility for these two individuals is variable expressivity of the mutation as it is passed down to further generations, increasing the number of teeth missing. However, because a genetic test for this condition is yet to be developed, none of these options can be confirmed with complete certainty.

There could be some implications both in terms of dentistry and the future of those affected. Agenesis of third molars could be beneficial in that they will not influence relapse following orthodontic treatment, or put the patient at risk of impaction and need for oral surgery. There may not be any disadvantages in terms of dentistry, however if these genes can be passed on, those involved may have some considerations. If this mutation only affects the third molars, there would be little to worry about in passing down the mutation. However, if this mutation has the potential to cause other teeth to be missing, it may be important for those affected to know.

\section{Conclusion}

These two individuals exhibit a very common dental abnormality that is most likely caused by an autosomal dominant mutation. There is little reason to be con- 
cerned for the future generations, unless there is also an association with impairment of overall health.

\section{References}

1. Genetic basis of dental agenesis--molecular genetics patterning clinical dentistry. Chhabra N, Goswami M, Chhabra A. Med Oral Patol Oral Cir Bucal. 2014 Mar 1;19(2):e112-9. Review. PMID: 24121910

2. The genetic basis of tooth development and dental defects. Thesleff I. Am J Med Genet A. 2006 Dec 1;140(23):2530-5. Review. PMID: 16838332

3. Association between polymorphism in the promoter region (G/C-915) of PAX9 gene and third molar agenesis. Bianch FJ, de Oliveira TF, Saito CB, Peres RC, Line SR. J Appl Oral Sci. 2007 Oct;15(5):382-6.

PMID: 19089165

4. Vieira, Alexandre R (May 2012) Genetics of Congenital Tooth Agenesis. eLS. John Wiley \& Sons, Ltd:Chichester. DOI:

10.1002/9780470015902.a 0023576 